\title{
A rheological constitutive model for semiconcentrated rod suspensions in Bingham fluids
}

\author{
J. Férec, ${ }^{1, a)}$ E. Bertevas, ${ }^{2}$ B. C. Khoo, ${ }^{2}$ G. Ausias, ${ }^{1}$ and N. Phan-Thien ${ }^{2}$ \\ ${ }^{1}$ Institut de Recherche Dupuy de Lôme (IRDL), Université Bretagne Sud, FRE CNRS 3744, IRDL, \\ F-56100 Lorient, France \\ ${ }^{2}$ Department of Mechanical Engineering, National University of Singapore, Singapore 119260, Singapore
}

(Received 31 March 2017; accepted 5 July 2017; published online 27 July 2017)

\begin{abstract}
A rheological constitutive law is developed for a suspension of rigid rods in a Bingham fluid for volume fractions ranging up to the semiconcentrated regime. Based on a cell model approach, which allows expressing the shear stress on the particle surface, the particle stress contribution is derived and involves additional yield stress terms related to an ensemble average orientation distribution of the rods. As a first approach, a von Mises criterion is used to describe the composite flow threshold, which is found to be anisotropic in the sense that it depends on the rod orientation. A rod dynamics equation is also proposed and incorporates some diffusion/perturbation due to yielded regions encountered throughout the suspension. In parallel, an equivalent kinetic theory is also developed. The model provides good agreement with shear stress experiments for kaolin pastes filled with steel fibers of two different aspect ratios. Published by AIP Publishing. [http://dx.doi.org/10.1063/1.4995436]
\end{abstract}

\section{INTRODUCTION}

The modeling of suspension rheology is a topic of much interest due to the wide range of applications of such systems in various areas of engineering. Fluids encountered in real-life situations generally exhibit complex behavior and the addition of particles amplifies this complexity. Although rather simplistic, the solid and fluid terms are still used to classify the ability of a material to flow when subjected to an external stress. Certain materials can flow when the applied stress is large enough but behave like a solid when this stress is small. The stress threshold between these two regimes is called the yield stress and the simplest model known to describe this behavior is the Bingham model, solely defined by a plastic viscosity and a yield stress. When particles are added to a purely viscous fluid, the suspension may exhibit a Bingham behavior. The existence of a yield stress depends on the volume fraction of particles, their geometry, and possible interactions between them. When increasing volume fraction, frictional and short-range forces between particles become large enough to create a network with solid-like behavior. This network can be destroyed if the applied stress exceeds the yield stress and the suspension then exhibits a fluid-like behavior. When added to a molten thermoplastic, a volume fraction of about $10 \%$ of microscale, high aspect ratio fibers is necessary in order to induce the appearance of a yield stress, ${ }^{1}$ whereas only a few percent of nanotubes lead to a solid-like behavior. ${ }^{2}$ At the red blood cell scale, plasma filled with microscale elements is considered as a homogeneous fluid and can be modeled by a Bingham model. ${ }^{3}$ For a cement paste containing fibers, it is observed that the relative yield stress increases with the relative packing fraction. ${ }^{4}$ Ouari et al. ${ }^{5}$ found that the yield stress was affected

\footnotetext{
a)Electronic mail: julien.ferec@univ-ubs.fr
}

at smaller fiber contents than expected. Hence, it is desirable to understand the behavior of a Bingham fluid when modified with particles.

Building materials are increasingly filled with fibers in order to improve their stiffness and reduce cracks into the material after solidification. These materials can be considered as a combination of a yield stress fluid filled with fibers. Since in this case the suspending fluid is itself a yield stress fluid, it is interesting to understand how the addition of fibers modifies the behavior of Bingham fluids. Thus, cement pastes can be considered as homogeneous at microscale, ${ }^{6}$ when short fibers are introduced to reinforce the material. Numerous papers describe the rheological behavior and the flow of these materials in terms of experiments and numerical simulations. A slump test is often used to characterize building materials. Ferrara et al. ${ }^{7,8}$ proposed a model for a self-compacting concrete filled with steel fibers and applied it to model the slump test. To test this model, numerical simulations have been developed using a smooth particle hydrodynamics (SPH) method ${ }^{9,10}$ or lattice Boltzmann approaches, ${ }^{11}$ in which the composite is assumed to follow a Bingham model. The other rheological tests used are compression tests or rotational rheometry in vane geometries. Chalencon et al. ${ }^{12}$ performed compression tests on fiber-filled mortar with various fiber volume fractions and aspect ratios. They developed a model taking into account properties of the matrix and fiber orientation statistics. Derakhshandeh et al. ${ }^{13,14}$ used a vane rheometer to characterize the behavior of pulp suspensions exhibiting Bingham behavior and determined the rheological coefficients of the Herschel-Bulkley model for different fiber volume fractions. It was shown that both the yield stress and the consistency viscosity increase with volume fraction while the power law index decreases. Finally, the modeling of fiber-filled concrete flows has also been investigated. Kang and $\mathrm{Kim}^{15}$ simulated the flow of a fiber-filled concrete in a molding process and 
analyzed fiber orientation evolution using the Jeffery model. ${ }^{16}$ Fiber orientation has been improved in the pipe extrusion process, ${ }^{17}$ which has been applied to the fabrication of concrete pipes reinforced with steel fibers. ${ }^{18}$ Zhou and $\mathrm{Li}^{19}$ used the ram extrusion to study fiber reinforced cementitious materials. In their work, a numerical simulation using the Herschel-Bulkley model was developed to model the process.

Most models developed for fiber suspensions assume a Newtonian fluid matrix. The pioneering work of Jeffery ${ }^{16}$ on the dynamics of fiber suspensions was followed by those of Batchelor, ${ }^{20}$ Hinch and Leal, ${ }^{21}$ and many others, but these studies were restricted to dilute suspensions. Later, Dinh and Armstrong ${ }^{22}$ developed a model accounting for interactions amongst randomly oriented fibers immersed in a Newtonian fluid. Following Jeffery, ${ }^{16}$ Hand, ${ }^{23}$ and Giesekus, ${ }^{24}$ Lipscomb et al. ${ }^{25}$ proposed a constitutive equation for dilute suspensions of ellipsoidal particles with large aspect ratio. Note that certain models have been developed for power-law or viscoelastic matrices. Gibson and Toll ${ }^{26}$ extended the theory of Dinh and Armstrong $^{22}$ to propose a model for the fiber dynamics with a non-Newtonian matrix. Souloumiac and Vincent ${ }^{27}$ established a stress expression that takes into account the shear-thinning behavior of the matrix, represented by a power-law viscosity. They determined the contribution of the fibers to the stress tensor using a cell model ${ }^{28}$ and assumed that hydrodynamic interactions between the fibers are weak. The effect of the fluid viscoelastic properties on rod dynamics was first investigated by Leal ${ }^{29}$ and Brunn ${ }^{30}$ using second-order fluids. These works allow for the development of a model for the fiber dynamics, which is impacted by the fluid viscoelasticity. Recently, Kagarise $^{31}$ investigated the rheology of nanofiber-filled molten polystyrene and they proposed a microstructure-based constitutive model, which predicts the rheological behavior observed experimentally and the evolution of nanofiber orientation well. The addition of non-spherical particles into a matrix leads to a material with non-isotropic behavior. Thus, White et $a{ }^{32}$ and Robinson et al. ${ }^{33}$ developed a model for a transversely isotropic viscoplastic fluid. Finally, a model has been proposed by Férec et al. ${ }^{34}$ for suspensions of rigid fibers in a non-Newtonian fluid exhibiting a yield stress by taking into account hydrodynamic and fiber-fiber interactions.

The purpose of this paper is to derive a constitutive equation for rod suspensions in fluids that exhibit a yield stress, in particular Bingham fluids, by using an approach based on the cell model. Following Batchelor ${ }^{28}$ and Goddard, ${ }^{35,36}$ who considered the stress generated in a non-dilute suspension of rods by pure straining motion for Newtonian and power-law fluids, respectively, we first determine the contribution to the macroscopic stress due to the presence of elongated rods in a Bingham liquid. Then, the yield surface in terms of rod orientations is investigated. In order to validate the model, a comparison is performed on kaolin pastes filled with steel fibers. Finally, a rod dynamics equation is proposed.

\section{DEFINITIONS AND HYPOTHESES}

The rod is represented by a straight cylinder of length $L$ having a circular cross section of radius $R$ (or equiva- lently of diameter $D$ ) and is assumed to be a slender body. Its large aspect ratio, defined by $a_{r}=L / D \gg 1$, allows us to neglect the particle end-effects. The cell model consists in coaxially embedding a test rod into a cylindrical fluid cell of radius $h$ and of the same length as the particle (Fig. 1). It is assumed that the effect of one particle on its neighbors can be approximated by an equivalent cylindrical boundary around the test rod and therefore simplifies the problem to a single-particle theory. Rod interactions are taken into account by solving a boundary value problem in the annular region, between the rod and the outer envelope. A velocity gradient results from this boundary-value problem that aims at modeling an interparticle interaction by conceptually restricting the fibre dynamics to a reptation-like motion in a constraining space. A dimensional analysis with the assumption of inertialess motion suggests that a quasi-steady and sheardominated flow occurs in the near field of the slender particle; this implies the validity of a viscometric flow representation for the fluid rheology. ${ }^{28,35-37}$ The rod suspension is assumed to be subjected to an unperturbed linearly varying bulk flow defined by $\boldsymbol{u}=\boldsymbol{\kappa} \cdot \boldsymbol{r}$, where $\boldsymbol{\kappa}$ is the transpose of the velocity gradient tensor ${ }^{38}$ and $\boldsymbol{r}$ is the position vector. The fluid motion is considered steady and Brownian motion effects are neglected.

One manner in which rod suspensions can be characterized is via concentration regimes. ${ }^{22} \mathrm{~A}$ rod suspension is said to be dilute if on average, less than one rod is found in a spherical volume of diameter equal to the rod length, i.e., $1 / a_{r}^{2}<\phi$, where $\phi$ is the volume fraction of rods. On the contrary, if the spacing between rods is $O(D)$, then the particle motion is hindered. Doi and Edwards ${ }^{39,40}$ showed that $\phi \approx O\left(1 / a_{r}\right)$ for a suspension of randomly oriented rods where the average distance between particles is $D$. When all rods are parallel to one another, the average spacing between them reduces and therefore enhances the volume fraction up to $\phi \approx O(1)$. Thus, the concentration limits of interest in this work are given in terms of the rod factor, $\phi a_{r}$, such that $1 / a_{r}<\phi a_{r}<1$ for random rods and $1 / a_{r}<\phi a_{r}<a_{r}$ for aligned rods.

Among the various models of viscoplastic fluids, ${ }^{41}$ which constitute an important class of non-Newtonian materials, the Bingham plastic model ${ }^{42}$ appears to be one of the simplest. In $3 \mathrm{D}$, the constitutive equation for a Bingham fluid takes the form $^{43}$

$$
\begin{aligned}
& \tau=\frac{\tau_{0}}{|\dot{\gamma}|} \dot{\gamma}+\eta_{B} \dot{\gamma} \text { for }|\tau|>\tau_{0}, \\
& \dot{\gamma}=\mathbf{0} \text { for }|\tau| \leq \tau_{0} .
\end{aligned}
$$

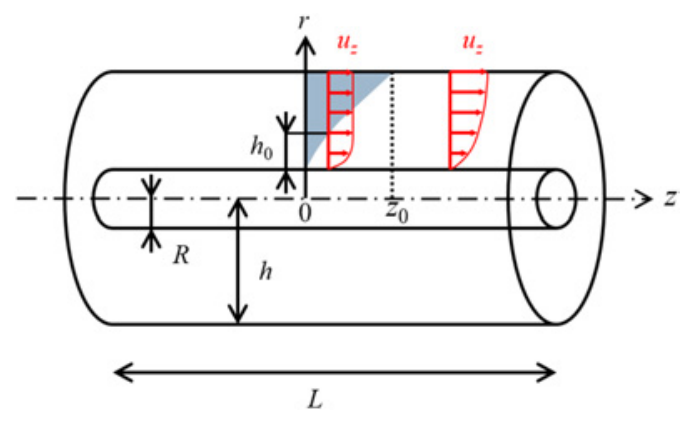

FIG. 1. Geometry of the cell surrounding the test rod. 
In the above, $\tau_{0}$ is the yield stress and $\eta_{B}$ is a constant plastic viscosity. $|\dot{\gamma}|$ represents the effective deformation rate of the strain rate tensor $\dot{\gamma}\left(\dot{\gamma}=\boldsymbol{\kappa}+\boldsymbol{\kappa}^{t}\right.$, where the superscript $t$ denotes the transpose operator), which is given by $|\dot{\gamma}|$ $=\sqrt{\frac{1}{2} \dot{\gamma}: \dot{\gamma}}$ (i.e., equal to the shear rate in a simple shear flow). Similarly, $|\tau|$ is the magnitude of the extra stress tensor $\tau$. An unyielded zone is formed when the stress $|\tau|$ falls below $\tau_{0}$.

\section{CELL MODEL APPROACH}

\section{A. Velocity profiles through the cell}

The cell model provides a useful approximation in the particle near field, with constant velocity gradients along the rod. ${ }^{28,35-37}$ Hence for this axisymmetric problem, the axial velocity $u_{z}$ satisfies the following equation of motion in terms of stress in cylindrical coordinates $(r, \theta$, and $z)$ :

$$
\frac{1}{r} \frac{\partial}{\partial r}\left(r \tau_{r z}\right)=0,
$$

where $\tau_{r z}$ represents the shear stress. If a Bingham model with a viscosity $\eta_{B}$ and a yield stress $\tau_{0}$ is used to characterize the rheological behavior of the fluid, such as the one given in Eq. (1a), Eq. (2) can be integrated twice to yield

$$
u_{z}=\frac{A}{\eta_{B}} \log (r)-\frac{\tau_{0}}{\eta_{B}} r+B
$$

where $A$ and $B$ are two constants of integration and are determined with the assignment of two velocity boundary conditions. At the rod surface, a no-slip condition is presumed, that is, $u_{z}(R)=0$, whereas at $r=h$ a relative fluid velocity with respect to the one of the particle is introduced. More precisely, the fluid velocity at a distance $z \mathbf{p}$ from the center of mass of the $\operatorname{rod}$ is $z \boldsymbol{\kappa} \cdot \mathbf{p}$ (where $\mathbf{p}$ is a unit vector directed along the main particle axis) and the rod velocity at the same location is $z \dot{p}$, which is normal to $\mathbf{p}$. Hence, the relative velocity component along the $z$-direction is found to be $u_{z}(h)=z \dot{\gamma}: \mathbf{p p} / 2$. As the boundary conditions are fixed in terms of velocities, flow may occur and thus the axial velocity $u_{z}$ will exhibit a Newtonian velocity profile in the yielded region, followed by a plug-flow in the unyielded region. The yield radius, $h_{0}$, is introduced to separate the regions where the yield stress has exceeded or not. In Fig. 1, the shaded area represents the unyielded region in which a plug-flow exists. Therefore, two velocity profiles have to be distinguished:

- Case (a) deals with a flow where the unyielded region is outside the cell, that is, $h_{0} \geq h$ (Fig. 1). By applying the above velocity boundary conditions, the axial velocity is found to be

$$
\begin{aligned}
u_{z}= & \frac{z}{2} \dot{\gamma}: \mathbf{p p} \frac{\log (r / R)}{\log (h / R)}+\frac{\tau_{0}}{\eta_{B}} \frac{\log (r / R)}{\log (h / R)}(h-R) \\
& -\frac{\tau_{0}}{\eta_{B}}(r-R) .
\end{aligned}
$$

Obviously when the yield stress goes to zero, the above result simplifies to the expression given for an effective Newtonian medium. ${ }^{37}$

- Case (b) involves a plug-flow in the region inside the cell where the yield stress is not exceeded $\left(h_{0}<r<h\right)$.
Here, analytical solutions for the velocity distribution are given by

$$
\begin{aligned}
u_{z}= & \frac{z}{2} \dot{\gamma}: \mathbf{p p} \frac{\log (r / R)}{\log \left(h_{0} / R\right)}+\frac{\tau_{0}}{\eta_{B}} \frac{\log (r / R)}{\log \left(h_{0} / R\right)}\left(h_{0}-R\right) \\
& -\frac{\tau_{0}}{\eta_{B}}(r-R) \text { for } \quad R \leq r<h_{0}, \\
u_{z}= & \frac{z}{2} \dot{\gamma}: \mathbf{p p} \text { for } h_{0} \leq r \leq h .
\end{aligned}
$$

The expression for $h_{0}$ is found by setting the velocity gradient to zero at $r=h_{0}$ in Eq. (5a), which yields after a long but straightforward calculation

$$
h_{0}(z)=R \exp \left[1+W_{0}(\chi(z))\right] .
$$

In the above, $W_{0}$ is the single-valued function (the principal branch) of the Lambert $W$-function. ${ }^{44} \chi(z)$ is given by $\chi(z)=a z+b$, in which $a=\left(\eta_{B}|\dot{\gamma}: \mathbf{p p}|\right) /\left(2 e \tau_{0} R\right)$ and $b$ $=-1 / e$, where $e=\exp (1)$ represents the Euler number. As the principal branch of the Lambert $W$-function is defined for $\chi(z) \geq-1 / e$, an absolute value for $\dot{\gamma}: \mathbf{p p}$ is imposed in the expression for $a$. By setting $h_{0}=h$ in Eq. (6), one can define a limiting abscissa, $z_{0}$, which separates cases (a) and (b) (see Fig. 1) and whose calculation leads to the following relation:

$$
z_{0}=\frac{2 R}{|\tilde{\gamma}: \mathbf{p p}|} B_{n}\left[1+\frac{h}{R}(\log (h / R)-1)\right],
$$

where $\tilde{\gamma}$ is the dimensionless strain-rate tensor. The Bingham number $B_{n}=\tau_{0} / \eta_{B}|\dot{\gamma}|$, representing the importance of the yield stress relative to viscous stress, is also introduced and depending on its value, three flow regimes can be defined as shown in Fig. 2.

\section{B. Drag force determination}

The shear stress on the rod surface, $\tau_{R}$, is obtained by taking the derivative for the axial velocity in Eqs. (4) and (5a) with respect to $r$ and then evaluating the stress using the constitutive law for a Bingham fluid. It is found that

$$
\left.\tau_{R}\right|_{(a)}=\frac{z}{2} \dot{\gamma}: \mathbf{p p} \frac{\eta_{B}}{R \log (h / R)}+\frac{\tau_{0}(h-R)}{R \log (h / R)}
$$

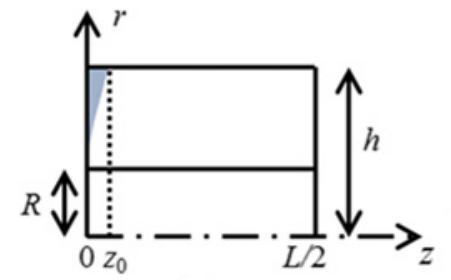

(a)

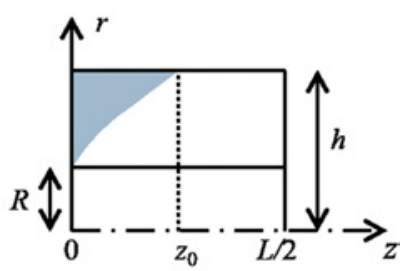

(b)

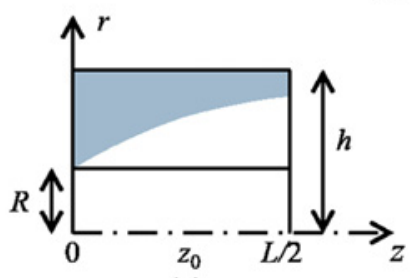

(c)

FIG. 2. Different flow regimes inside the cell. The shaded region represents the unyielded zone. (a) $B_{n} \ll 1$. (b) $B_{n} \approx 1$. (c) $B_{n} \gg 1$. 
for case (a) and

$$
\left.\tau_{R}\right|_{(b)}=\frac{z}{2} \dot{\gamma}: \mathbf{p p} \frac{\eta_{B}}{R \log \left(h_{0} / R\right)}+\frac{\tau_{0}\left(h_{0}-R\right)}{R \log \left(h_{0} / R\right)}
$$

for case (b). Both results are similar, except that case (a) involves $h$ and case (b) requires $h_{0}$, which we recall to be a function of $z$. Note that the $z$-dependency for $h_{0}$, represented by the bracketed $z$ in Eq. (6), has been removed for clarity. Finally, an elementary force balance on the particle leads to the drag force per unit length $f(z)=2 \pi R \tau_{R}$ for both cases.

\section{TOTAL STRESS TENSOR}

Constitutive equations for rod-filled systems may generally be written by considering them as two-component fluids, in which the total stress in the composite is assumed to be

$$
\sigma=-P \delta+\tau^{m}+\tau^{p} .
$$

In the above equation, $P$ is the hydrostatic pressure and $\delta$ is the identity tensor. $\tau^{m}$ represents the matrix contribution, which is given by Eq. (1) and the particle contribution to the extra stress tensor, $\tau^{p}$, remains to be expressed.

\section{A. Particle stress contribution}

The Kramer expression ${ }^{45}$ is used to derive the rod stress tensor, in which the volume fraction of particles, $\phi$, takes into account the contribution of each particle contained in a given volume. Hence, the extra stress due to the particle contribution is

$$
\boldsymbol{\tau}^{p}=\frac{\phi}{V}\left\langle\mathbf{p} \int_{z=-L / 2}^{z=L / 2} \mathbf{f}(z) d z\right\rangle,
$$

where $V=\pi R^{2} L$ represents the rod volume and the angular brackets denote the ensemble average with respect to the distribution function of $\mathbf{p}$. Batchelor ${ }^{28}$ argued that the tension force exerted by the fluid, $\mathbf{f}(z)$, is parallel to the unit vector $\mathbf{p}$ as the components normal to the rod make no contribution to the stress in the case of a particle on which no external force or couple act. Hence, $\mathbf{f}(z)=\bar{f}(z) \mathbf{p}$, where the tension magnitude, $\bar{f}(z)$, is related to the drag force, $f(z)$, by $\bar{f}(z)=-\int_{z=-L / 2}^{z} f(z) d z$. The above results are then substituted into Eq. (11) and after application of the integration by parts formula, the following expression is obtained:

$$
\tau^{p}=\frac{2 \phi}{\pi R^{2} L}\left\langle\mathbf{p p} \int_{z=0}^{z=L / 2} z f(z) d z\right\rangle .
$$

To derive Eq. (12), note that the integration in Eq. (11) can be performed over the half length of the rod due to the problem symmetry and the fact that only the hydrodynamic force applied all along the rod length has been used, that is, $\int_{z=-L / 2}^{z=L / 2} f(z) d z=0$. Finally, the rod stress tensor has to be expressed for the three cases depicted in Fig. 2.

\section{B. $B_{n} \ll 1$}

For $B_{n} \ll 1$, the unyielded region inside the cell can be neglected, leading us to consider the velocity field given by Eq. (4). Substitution of the drag force derived from the shear stress on the rod surface into Eq. (12) yields

$$
\boldsymbol{\tau}^{p}=\frac{2 \phi}{\pi R^{2} L}\left\langle\mathbf{p p} \int_{z=0}^{z=L / 2} z\left(\left.2 \pi R \tau_{R}\right|_{(a)}\right) d z\right\rangle .
$$

Integration can be performed very simply and results in

$$
\tau^{p}=\eta_{B} \phi \frac{a_{r}^{2}}{3 \log (h / R)} \dot{\gamma}:\langle\mathbf{p p p p}\rangle+\tau_{0} \phi \frac{a_{r}(h / R-1)}{\log (h / R)}\langle\mathbf{p p}\rangle,
$$

where terms in angular brackets represent the well-known orientation tensors ${ }^{46}$ and describe the rod orientation statistics in a representative elementary volume in an efficient and concise way.

In order to tackle cases in dilute regimes (i.e., $\phi<1 / a_{r}^{2}$ ), Batchelor ${ }^{28}$ proposed that the cell radius $h$ can be replaced by $L$, the rod length, which corresponds to allowing a particle to rotate freely without encountering any neighbor. Under this assumption, coupling coefficients in front of $\langle\mathbf{p p p p}\rangle$ and $\langle\mathbf{p p}\rangle$ are given by $\eta_{B} \phi a_{r}^{2} / 3 \log \left(2 a_{r}\right)$ and $\tau_{0} \phi a_{r}\left(2 a_{r}\right.$ $-1) / \log \left(2 a_{r}\right)$, respectively. In their theory for semiconcentrated fiber suspensions, Dinh and Armstrong ${ }^{22}$ related the ratio $h / R$ to the average lateral spacing between the particles. Two estimates for $h / R$ are available in the literature ${ }^{39,40}$ depending on the rod orientation state: $h / R=\sqrt{\pi / \phi}$ is obtained by considering that the rods are all parallel and fully aligned while $h / R=\pi /\left(2 \phi a_{r}\right)$ assumes a random orientation. Note that the last term in Eq. (14) points out that the yield stress of the suspending matrix induces an additional yield stress to the particle stress contribution. Therefore, the total stress tensor for the composite is found to be

$$
\begin{aligned}
\boldsymbol{\sigma}= & -P \boldsymbol{\delta}+\frac{\tau_{0}}{|\dot{\gamma}|} \dot{\gamma}+\eta_{B} \dot{\gamma}+\eta_{B} \phi \frac{a_{r}^{2}}{3 \log (h / R)} \dot{\gamma}:\langle\mathbf{p p p p}\rangle \\
& +\tau_{0} \phi \frac{a_{r}(h / R-1)}{\log (h / R)}\langle\mathbf{p p}\rangle \text { for }|\tau|>\left|\tau_{y}\right|, \\
\dot{\boldsymbol{\gamma}}= & \mathbf{0} \text { for }|\tau| \leq\left|\tau_{y}\right| .
\end{aligned}
$$

Equation (15a) represents a rheological constitutive equation for slender rods in Bingham fluids and reduces to the expression of Dinh and Armstrong 22 obtained for semiconcentrated fiber suspensions in Newtonian fluids if the yield stress, $\tau_{0}$, is set to zero. It is interesting to note that the yield stress involves two contributions (terms proportional to $\tau_{0}$ ): the standard one comes from the unfilled matrix, i.e., the second term in Eq. (15a), and an additional part due the presence of the rods, i.e., the last term in Eq. (15a). Moreover, this last expression is directly related to the particle orientation state, $\langle\mathbf{p p}\rangle$, and therefore leads to an anisotropic effective yield stress. To the best of our knowledge, it is the first time that this kind of result is derived. If the yield stress contribution is presumed to be independent of the hydrostatic pressure, it is given by

$$
\boldsymbol{\tau}_{y}=\frac{\tau_{0}}{|\dot{\gamma}|} \dot{\gamma}+\tau_{0} \phi \frac{a_{r}(h / R-1)}{\log (h / R)}\langle\mathbf{p p}\rangle .
$$

For weak flows, where the velocity gradient is $O(\epsilon)$, it is possible to approximate the second-order orientation tensor. Based on the work of Hinch and Leal, ${ }^{47}$ we can derive the following expression for the yield stress:

$$
\tau_{y}=\frac{\tau_{0}}{|\dot{\gamma}|} \dot{\gamma}+\eta_{B} \phi \frac{a_{r}^{2}}{15 \log (h / R)} \dot{\gamma} .
$$


In order to investigate the effect of rod orientation on the yield surface, we consider the response under a shearing/elongational flow as follows:

$$
\dot{\gamma}=\left[\begin{array}{ccc}
2 \dot{\epsilon} & \dot{\gamma} & 0 \\
\dot{\gamma} & -\dot{\epsilon} & 0 \\
0 & 0 & -\dot{\epsilon}
\end{array}\right]_{\left(\mathbf{e}_{1} \mathbf{e}_{2} \mathbf{e}_{3}\right)},
$$

which superimposes a simple shear flow in the 1-2 plane with a uniaxial elongation in the 1-direction (for $\dot{\epsilon}>0$ ) or a biaxial stretching flow $(\dot{\epsilon}<0)$, where $\dot{\gamma}$ and $\dot{\epsilon}$ represent the shear and elongation rates, respectively. The anisotropy parameters that are the components of the orientation tensor, $\langle\mathbf{p p}\rangle$, are material constants and relate to a fixed degree of anisotropy. As further discussed below, these scalars need to be considered as state variables each having an evolutionary equation coupled with the flow equations and ranging between two limiting orientation states (3D random and fully aligned rod orientations). Different yield criteria have been used, ${ }^{48}$ the most common for 3D constitutive relation being the von Mises criterion, $\left|\tau_{y}\right|$, which predicts that the material flows and deforms significantly only when the second invariant of the stress tensor exceeds the yield stress, $\left|\tau_{y}\right|=\sqrt{\frac{1}{2}\left(\tau_{y}: \tau_{y}\right)}$; otherwise the material behaves like a strained solid. Application of an anisotropic criterion is left as a topic of future research.

Figure 3 depicts the threshold curves with varying degrees of anisotropy (i.e., different rod orientation states). For a suspension with a 3D isotropic distribution of rods (Iso.), that is, $\langle\mathbf{p p}\rangle=\boldsymbol{\delta} / 3$, it is not surprising that the size of the elastic domain increases monotonically in contrast with the unfilled matrix (Mat.). Perfect alignment of rods with the 1-direction (Ali.), which corresponds to the stretching direction for the uniaxial elongational flow, leads to $\langle\mathbf{p p}\rangle=\mathbf{e}_{1} \mathbf{e}_{1}$. The threshold curve exhibits a significant anisotropy, which cannot be described by a quadratic criterion. Yield stress maxima are observed when the uniaxial elongational flow field is dominant and minima occur for a prevalent biaxial flow field. For a simple shear flow, it can be observed that the yield stress value is significantly larger with a perfect alignment of rods

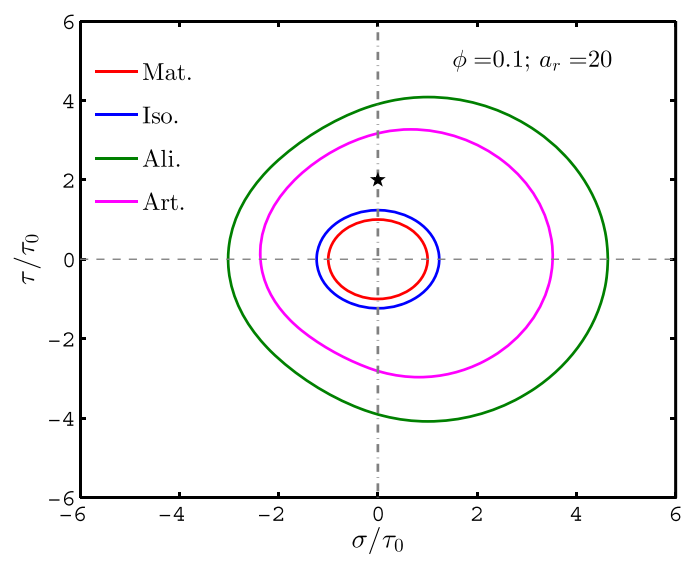

FIG. 3. Dimensionless yield surface for different systems: unfilled matrix (Mat.) and suspensions with isotropic (Iso.), aligned (Ali.), and artificial (Art.) rod orientation states. A simple shear flow is superimposed with a biaxial stretching flow on the left side of the vertical axis, whereas a simple shear flow is superimposed with a uniaxial elongation flow on the right side. along the shear flow direction (Ali.) when compared with an isotropic rod orientation state (Iso.). This may be explained by considering the average distance between rods, $h$, for these both orientation states. Following the definition given previously, it results that the inverse of $h$ for a random orientation state is larger than the one for a perfect alignment of rods. According to Eq. (2), the local shear stress, $\tau_{r z}$, is found to be proportional to $1 / r$, the inverse of a distance. If this length is now replaced by $h$, it results that the local shear stress for an aligned configuration is lower than the one for a random orientation state. Hence, the flow threshold will first be exceeded for a random rod configuration, explaining the observed model prediction that the yield stress value is the largest for a perfect alignment of rods along the shear flow direction. Furthermore, under transient conditions where the rods may initially be randomly orientated and convected with the flow, this suggests that the yield surface will evolve and therefore the suspension can stop flowing (see Subsection V C). Finally, for the following artificial rod distribution state (Art.):

$$
\langle\mathbf{p p}\rangle=\left[\begin{array}{ccc}
0.7 & 0.1 & 0 \\
0.1 & 0.2 & 0 \\
0 & 0 & 0.1
\end{array}\right]_{\left(\mathbf{e}_{1} \mathbf{e}_{2} \mathbf{e}_{3}\right)},
$$

it is found that the threshold curve lies between the two limits for the rod orientation distribution.

The influence of the rod aspect ratio, $a_{r}$, the particle volume fraction, $\phi$, and more precisely of the rod factor, $\phi a_{r}$, on the dimensionless shear yield stress is illustrated in Fig. 4. The vertical dashed-dotted lines denote the limits of validity of the theory for both limits of the orientation distributions. Note that a differentiation is made between the two curves (Ali. $a_{r}=20$ and Ali. $\phi=0.1$ ): the first one considers a constant aspect ratio of 20 , whereas the second one assumes a fixed value of 0.1 for the particle volume fraction. An asymptotic analysis for large aspect ratios leads to



FIG. 4. Dimensionless shear yield stress as a function of the rod factor for different rod distributions: isotropic (Iso.), aligned in the shear flow direction with a constant aspect ratio (Ali. $\left.a_{r}=20\right)$ and aligned in the shear flow direction with a constant particle volume fraction (Ali. $\phi=0.1$ ). The vertical dasheddotted lines denote the limits of validity of the theory for random and aligned orientation distributions. 


$$
\begin{aligned}
\tau^{I s o} & =\tau_{0} \phi \frac{\left(a_{r}-\pi / 2 \phi\right)}{\sqrt{6}\left|\log \left(\pi / 2 \phi a_{r}\right)\right|}+O\left(1 / a_{r}\right), \\
\tau^{A l i} & =\tau_{0} \phi \frac{\sqrt{2} a_{r}(\sqrt{\pi / \phi}-1)}{\log (\pi / \phi)}+O\left(1 / a_{r}\right),
\end{aligned}
$$

which is also reported in Fig. 4.

It is now interesting to test the proposed model against some experimental results. Fig. 5 depicts the dimensionless shear yield stresses for kaolin pastes filled with steel fibers of aspect ratios $a_{r}=37.5$ and $a_{r}=81$, respectively. The measured yield stress values of the unfilled kaolin pastes are found to be $404 \pm 32 \mathrm{~Pa}$ and $521 \pm 14 \mathrm{~Pa}$ for the first and second suspensions, respectively. The experimental results are taken from the work of Férec et al. ${ }^{34}$ and fiber orientation distribution is presumed to be isotropic for both systems. The model predictions for the two extreme orientation states (i.e., perfectly aligned and randomized rods) are also reported in Fig. 5 by considering the fiber properties. For the lowest rod factor values, good agreement is observed. Although the rod orientation for the systems are assumed to be isotropic, model predictions assuming a perfect alignment of the rods appear to be closer to the experimental data for cases involving larger rod factors. Unfortunately, the viscosity for kaolin pastes was not measured and the Bingham number for both systems

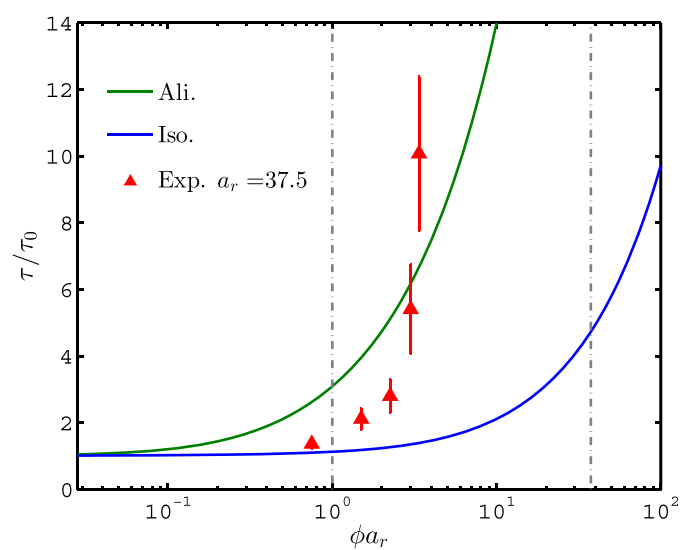

(a)

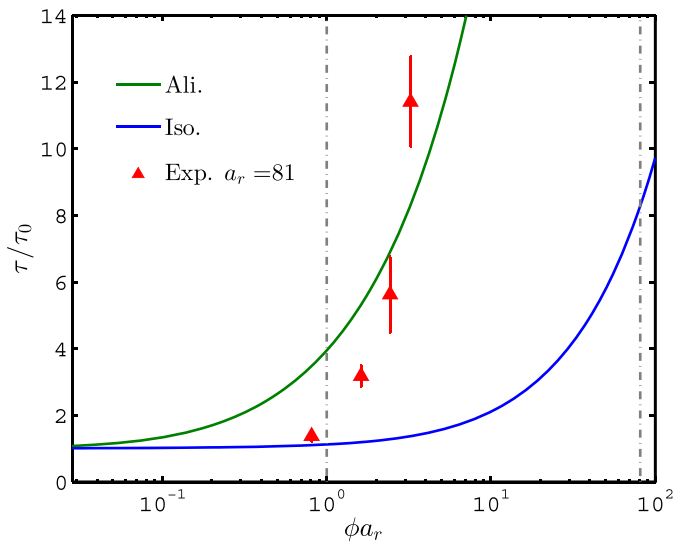

(b)

FIG. 5. Dimensionless shear yield stress as a function of the fiber factor: comparison between model predictions for the two extreme orientation distributions and experimental data. ${ }^{34}$ The vertical dashed-dotted lines denote the limits of validity of the theory for both limits of the orientation distributions. (a) Rods with $a_{r}=37: 5$. (b) Rods with $a_{r}=81$. cannot be evaluated; this could also explain the observed discrepancies. We remind the reader that the present theory is valid for $B_{n} \ll 1$.

\section{C. $B_{n} \approx 1$}

When $B_{n} \approx 1$, an unyielded region occurs inside the cell up to the abscissa $z_{0}$ but disappears before reaching the edge of the rod. In this context, the integration involved in Eq. (12) requires the use of the additivity rule on intervals and then the rod stress tensor obtained is

$$
\begin{aligned}
\boldsymbol{\tau}^{p}= & \frac{2 \phi}{\pi R^{2} L}\left\langle\mathbf{p p} \int_{z=0}^{z=z_{0}} z\left(\left.2 \pi R \tau_{R}\right|_{(b)}\right) d z\right. \\
& \left.+\mathbf{p p} \int_{z=z_{0}}^{z=L / 2} z\left(\left.2 \pi R \tau_{R}\right|_{(a)}\right) d z\right\rangle .
\end{aligned}
$$

Note that the first integrand involves $h_{0}$, which is a function of $z$. Therefore, after some long and tedious calculations (some details of which are found in Appendix A), Eq. (21) becomes

$$
\begin{aligned}
\boldsymbol{\tau}^{p}= & \eta_{B} \phi \frac{a_{r}^{2}}{3 \log (h / R)} \dot{\boldsymbol{\gamma}}:\langle\mathbf{p p p p}\rangle+\tau_{0} \phi \frac{a_{r}(h / R-1)}{\log (h / R)}\langle\mathbf{p p}\rangle \\
& +\tau_{0} \phi \frac{B_{n}^{2}}{a_{r}}\langle\Theta(\tilde{\gamma}, \mathbf{p}) \mathbf{p p}\rangle,
\end{aligned}
$$

where $\Theta(\tilde{\gamma}, \mathbf{p})$ is a function of the dimensionless strain-rate tensor, $\tilde{\gamma}$, and the unit vector directed along the main particle axis, p. When compared with Eq. (14), the last term proportional to $\Theta(\tilde{\gamma}, \mathbf{p})$ represents an additional contribution to the yield stress. The function $\Theta(\tilde{\gamma}, \mathbf{p})$ involves some indeterminate forms and therefore its resolution is left as a topic of future research. Nevertheless, pre-factors in the two yield stress contributions suggest that the last term in Eq. (22) may be negligible. Indeed, it is proportional to $1 / a_{r}$ as compared to $a_{r}$ for the other one. With this assumption, the above equation may reduce to Eq. (14) if components of $\langle\Theta(\tilde{\gamma}, \mathbf{p}) \mathbf{p p}\rangle$ are $O(1)$.

\section{D. $B_{n} \gg 1$}

It remains to deal with the case corresponding to $B_{n} \gg$ 1 , for which an unyielded region is observed throughout the whole cell. Hence, the shear stress at the rod surface given in Eq. (9) is used to express the drag force leading to the following extra stress tensor for the particle contribution:

$$
\boldsymbol{\tau}^{p}=\frac{2 \phi}{\pi R^{2} L}\left\langle\mathbf{p p} \int_{z=0}^{z=L / 2} z\left(\left.2 \pi R \tau_{R}\right|_{(b)}\right) d z\right\rangle .
$$

The calculations described in Appendix B result in the following extra stress due to the particle:

$$
\begin{aligned}
\tau^{p}= & \eta_{B} \phi \frac{a_{r}^{2}}{3 \log (h / R)} \dot{\gamma}:\langle\mathbf{p p p p}\rangle+\tau_{0} \phi \frac{a_{r}(h / R-1)}{\log (h / R)}\langle\mathbf{p p}\rangle \\
& +\tau_{0} \phi \frac{B_{n}^{2}}{a_{r}}\langle\Phi(\tilde{\gamma}, \mathbf{p}) \mathbf{p p}\rangle,
\end{aligned}
$$

where the function, $\Phi(\tilde{\gamma}, \mathbf{p})$, is once more related to the dimensionless strain-rate tensor and the unit vector directed along the rod. This function $\Phi$ differs from $\Theta$ but suffers from the same indeterminacy. We do not pursue this any further. 
Note however that the pre-factor to $\Phi$ is similar to the one of the second term on the right-hand side and is likely to be non-negligible, or at least of the same order as the second term.

\section{ROD ORIENTATION DYNAMICS}

\section{A. Expression for the evolution equation}

In order to derive the evolution equation for the rod microstructure described by the second-order orientation tensor $\langle\mathbf{p p}\rangle$, we make use of the ingenious method to determine the stress tensor proposed by Giesekus ${ }^{24}$ (which is frequently used in the kinetic theory of polymer solutions and melts ${ }^{45}$ ). Known as the Giesekus stress tensor, this result can be used to derive the extra stress due to the particle contribution and is given by

$$
\tau^{p}=-\frac{4 \phi \zeta}{\pi R^{2} L} \frac{\mathfrak{D}\langle\mathbf{p p}\rangle}{\mathfrak{D} t},
$$

where $\zeta$ is a drag coefficient and $\mathfrak{D} / \mathfrak{D} t$ denotes the convected derivative of the contravariant components of a second-order tensor, $\Delta$, such that

$$
\frac{\mathfrak{D} \Delta}{\mathfrak{D} t}=\frac{D \Delta}{D t}+\frac{1}{2}(\omega \cdot \Delta-\Delta \cdot \omega)-\frac{1}{2}(\dot{\gamma} \cdot \Delta+\Delta \cdot \dot{\gamma}),
$$

in which $\omega$ represents the vorticity tensor. Therefore, by equating Eqs. (14) and (25), the convected derivative for $\langle\mathbf{p p}\rangle$ (i.e., the time evolution for the rod orientation) can be expressed as

$$
\frac{\mathfrak{D}\langle\mathbf{p p}\rangle}{\mathfrak{D} t}=-\dot{\gamma}:\langle\mathbf{p p p p}\rangle-\tau_{0} \frac{3(h / R-1)}{a_{r} \eta_{B}}\langle\mathbf{p p}\rangle,
$$

and the drag coefficient is found to be

$$
\zeta=\frac{\eta_{B} \pi L^{3}}{3 \log (h / R)}
$$

It is well known that the trace of $\langle\mathbf{p p}\rangle$ must remain constant and equal to one, ${ }^{46}$ which is not guaranteed by Eq. (27) because the trace of the material derivative, $D\langle\mathbf{p p}\rangle / D t$, is not null. To overcome this inconsistency, a Lagrange multiplier is incorporated into Eq. (27) to enforce a spatial constraint related to the fact that rods are rigid and therefore cannot stretch. Thus, Eq. (27) becomes

$$
\frac{\mathfrak{D}\langle\mathbf{p p}\rangle}{\mathfrak{D} t}=-\dot{\gamma}:\langle\mathbf{p p p p}\rangle+\tau_{0} \frac{(h / R-1)}{a_{r} \eta_{B}}(\boldsymbol{\delta}-3\langle\mathbf{p p}\rangle) .
$$

The first term on the right-hand side of Eq. (29) stands for the hydrodynamic contribution, whereas the last part is a diffusion term related to the yield stress of the suspending fluid, $\tau_{0}$. This suggests that under flow, rods will tend to align along the streamlines but will be perturbed by some unyielded regions encountered throughout the suspension. This last part shares some similarities with the one found when taking into account particle interactions, except that in the present case, the proportionality term is not a function of the effective deformation rate. $^{49}$

In this form, Eq. (29) suggests that with no flow (i.e., $\dot{\gamma}=\mathbf{0}$ ), a randomizing effect will occur leading to a final isotropic orientation state for the rods. This is obviously impossible as the particles are considered non-Brownian; therefore, the equation for the rod orientation dynamics, Eq. (29), should be split into two contributions (similar to the definition for a Bingham fluid),

$$
\begin{gathered}
\frac{\mathfrak{D}\langle\mathbf{p} \mathbf{p}\rangle}{\mathfrak{D} t}=-\dot{\gamma}:\langle\mathbf{p p p p}\rangle+B_{n}|\dot{\gamma}| \frac{(h / R-1)}{a_{r}}(\boldsymbol{\delta}-3\langle\mathbf{p p}\rangle) \\
\text { for }|\tau|>\left|\tau_{y}\right|, \\
\frac{\mathfrak{D}\langle\mathbf{p} \mathbf{p}\rangle}{\mathfrak{D} t}=\mathbf{0} \text { for }|\tau| \leq\left|\tau_{y}\right| .
\end{gathered}
$$

Note that when the Bingham number is introduced, the effective deformation rate appears in the diffusive contribution, as illustrated in Eq. (30a).

\section{B. Equivalent kinetic theory}

The proposed model for rod dynamics is developed by means of moment-tensor equations, that is, at the macroscopic level, as typically used in process modeling. On the other hand, most models for rod suspensions are initially derived at the particle scale via the kinetic theory, which contains explicit expressions for a single rod evolution, and then provides a Fokker-Planck equation that describes the dynamics for a population of rods. Note however that obtaining numerical solutions for the Fokker-Planck equation is generally difficult and computationally expensive, and therefore, moments of the probability distribution function are statistical tools usually used to efficiently describe the microstructure properties. However, moment-tensor equations always require closure approximations, while kinetic theories do not. Thus, kinetic-theory equations provide important information for the development and validation of closure approximations. Hence, it is worth wondering if there is a kinetic theory that corresponds to the proposed model. Preliminary work shows that the following rod orientation evolution equation could answer this question (see Appendix $\mathrm{C}$ for details):

$$
\begin{gathered}
\frac{\mathfrak{D} \mathbf{p}}{\mathfrak{D} t}=-\frac{1}{2} \dot{\gamma}: \mathbf{p p p}-B_{n}|\dot{\gamma}| \frac{(h / R-1)}{2 a_{r} \psi_{\mathbf{p}}} \frac{\partial \psi_{\mathbf{p}}}{\partial \mathbf{p}} \\
\text { for }|\tau|>\left|\tau_{y}\right|, \\
\frac{\mathfrak{D} \mathbf{p}}{\mathfrak{D} t}=\mathbf{0} \text { for }|\tau| \leq\left|\tau_{y}\right|,
\end{gathered}
$$

where $\mathfrak{D} / \mathfrak{D} t$ denotes the convected derivative of the contravariant components of a vector and $\psi_{\mathbf{p}}$ is the probability distribution function from which the ensemble averages are performed. It is interesting to note that the first term in Eq. (31a) is the Jeffery equation expressed in terms of slender bodies and the last part leads to some diffusivity/perturbation induced by some unyielded regions encountered throughout the suspension.

\section{Orientation predictions in a transient shear flow}

The predictions for the orientation evolution have been calculated for a single point in simple shear for a sequence of creep tests. When compared with a stress growth experiment that consists in applying a constant shear rate, a creep test imposes a constant shear stress, which in terms of constitutive equations is more difficult to address. Indeed a minimization procedure must be used to find the optimum shear rate leading the desired shear stress for a given orientation before being 
subsequently updated. As discussed above, a closure approximation is necessary to express $\langle\mathbf{p p p p}\rangle$ in terms of $\langle\mathbf{p p}\rangle$ and can be done with the ORW3 closure approximation (orthotropic fitted closure approximation for wide interaction coefficient values with third-order polynomial expansions) developed by Chung and Kwon. ${ }^{50}$ Although a refined calculation could be made giving $h$ as a function of $\langle\mathbf{p p}\rangle$ (see, for example, Ref. 51), such a refinement is not investigated here and we simply set $h / R=\sqrt{\pi / \phi}$. The other parameters used for the simulation are arbitrarily chosen as $\eta_{B}=100 \mathrm{~Pa} \cdot \mathrm{s}, a_{r}=50, \phi=10 \%$, and $\tau_{0}=10 \mathrm{~Pa}$.

The test consists in applying three successive constant shear stress: $\sigma_{12}=60 \mathrm{~Pa}$ for $20 \mathrm{~s}$, then $\sigma_{12}=100 \mathrm{~Pa}$ for $100 \mathrm{~s}$, and finally, again $\sigma_{12}=60 \mathrm{~Pa}$ for $20 \mathrm{~s}$ as shown in Fig. 6(a). The initial value for the shear stress is chosen so that it is contained between the two extreme limits of the magnitude of the yield stress contribution, namely, for isotropic and aligned rod orientation states. With regards to Fig. 3 , this shear stress is symbolically represented by a pentagram on the vertical axis between the blue curve (i.e., isotropic orientation state) and the green curve (i.e., perfectly alignment orientation state). Before starting the flow simulation, the initial rod orientation is assumed to be isotropic.

For the first sequence, $0<t<20 \mathrm{~s}$, Fig. 6(b) reports the time evolution for the components $\left\langle p_{1} p_{1}\right\rangle,\left\langle p_{2} p_{2}\right\rangle$, and $\left\langle p_{1} p_{2}\right\rangle$, where subscripts 1 and 2 stand for flow and velocity gradient directions, respectively. It is observed that the initial random orientation is destroyed before remaining in a fixed state at a time value close to $14 \mathrm{~s}$. As reported in Fig. 6(a), the yield stress increases with rod orientation to ultimately join the shear stress and lead to the cessation of the microstructure evolution. In terms of Fig. 3, the location of the pentagram remains unchanged but the yield surface defined by the blue curve increases as the rod orientation until the boundary attains the pentagram. Figure 6(c) shows that the first and second normal stress differences go initially from zero to positive values until the cessation of the microstructure evolution. Thus, in this yielded state, the first normal stress difference exhibits a constant positive value, and a constant negative value is observed for the second normal stress difference. These non-zero normal stress differences arise from the last term in Eq. (16) as $\langle\mathbf{p p}\rangle$ is no longer isotropic.

For the second sequence comprised between 20 and $120 \mathrm{~s}$, the applied shear stress $\left(\sigma_{12}=100 \mathrm{~Pa}\right)$ is much higher than the yield stress value, as depicts in Fig. 6(a). When looking back to Fig. 3, this test sequence consists in moving the pentagram vertically in the positive direction beyond the green curve. Hence, a "standard" rod evolution starting from the previous orientation state is observed before reaching a steady-state [see Fig. 6(b)]. Figure 6(c) reports a large increase before reaching a steady-state for the first normal stress difference without exhibiting overshoot (at least, with this set of parameters) and a decrease to a constant negative value close to zero for the second normal stress difference.

Finally, the test finishes when the applied shear stress goes back to $60 \mathrm{~Pa}$ for $20 \mathrm{~s}$ [see Fig. 6(a)]. With the help of Fig. 3, the pentagram reaches a position inside both yield surfaces defined by the blue and green curves. Thus, a second yielded state is reached as the effective yield stress of the composite is higher than the applied shear stress, as depicted in Fig. 6(a). The internal microstructure defined by the rod orientation state remains unchanged, as shown in Fig. 6(b). As for both normal

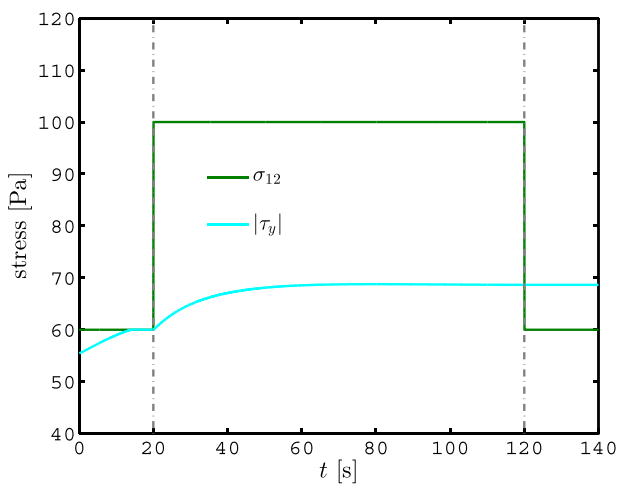

(a)

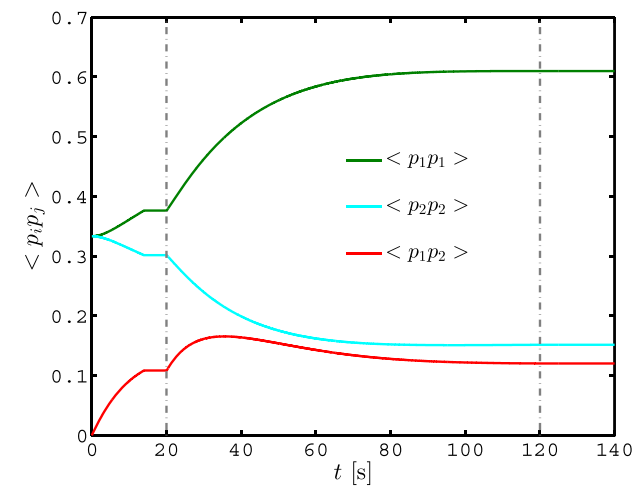

(b)



FIG. 6. System responses when applying a sequence of creep tests. (a) Sequence of applied shear stress with magnitudes of $\sigma_{12}=60,100$ and $60 \mathrm{~Pa}$, respectively. $\left|\tau_{y}\right|$ represents the magnitude of the yield stress contribution given by Eq. (16). (b) Orientation components as functions of time. (c) First and second normal stress differences as functions of time. 
stress differences reported in Fig. 6(c), they exhibit constant values, positive for $\sigma_{11}-\sigma_{22}$ and negative for $\sigma_{22}-\sigma_{33}$. Note that their magnitudes differ from the first yielded state as the rod orientation has evolved during the second sequence.

\section{CONCLUSION}

In this work, a cell model is used to derive a constitutive equation for rod suspensions in a Bingham fluid without introducing tuning parameters. Three expressions for the particle stress are proposed depending on the Bingham number. For Bingham numbers less than unity, a straightforward relationship is obtained in terms of the moments for the orientation distribution probability, known as the orientation tensors. It is found that the presence of rods leads to an increase in the yield stress values and more significantly, it is related to the average particle orientation. The effect of rod orientation on the yield stress surface is investigated by considering the response under a shear/elongational flow. It appears that the shear yield stress for randomly oriented rods is lower than the one for the case where all particles are aligned with the flow direction. The model predictions are tested against shear stress experiments for kaolin pastes filled with steel fibers having two different aspect ratios. Good agreement is observed since the model predictions give qualitative and quantitative bounds for yield stresses of both suspensions. For Bingham numbers close to and larger than one, some unresolved issues are encountered and suggest more specific works.

Particle dynamics in a Bingham fluid was also determined: the Jeffery solution expressed in terms of slender bodies (that is, $a_{r} \gg 1$ ), in which a diffusivity/perturbation term proportional to the yield stress of the unfilled matrix is introduced, is found to be suitable. Hence, a similar moment-tensor equation, which is useful at the macroscopic level, is obtained from the Giesekus stress tensor and from the kinetic-theory. It is also found that the rod microstructure will evolve with the flow if the applied stress exceeds the threshold stress and will otherwise remain unchanged. Since a kinetic-theory form of the proposed model is given, it can be used to perform closure-free calculations and to test closure approximations for its momenttensor form. As rod suspensions exhibit non-zero normal stress differences, the model is also able to predict yield surface for the first and second normal stress coefficients although this aspect of rod suspension behavior deserves more experimental investigation.

\section{APPENDIX A: INTEGRATION CALCULATIONS INVOLVED FOR $B_{n} \approx 1$}

Equation (21) involves two integrands, called $I$ and $J$, respectively, and are defined by

$$
I=\int_{z=0}^{z=z_{0}} z\left(\left.2 \pi R \tau_{R}\right|_{(b)}\right) d z
$$

and

$$
J=\int_{z=z_{0}}^{z=L / 2} z\left(\left.2 \pi R \tau_{R}\right|_{(a)}\right) d z
$$

First, our attention is focused on Eq. (A1), which involves the dependency of $h$ on $z$ and therefore requires some specific mathematical operations. Hence, by inserting the expression for the shear stress at the rod surface in Eq. (A1), I can be split into two sums, $I=I_{1}+I_{2}$, such that

$$
I_{1}=\pi \eta_{B} \dot{\gamma}: \mathbf{p p} \int_{z=0}^{z=z_{0}} \frac{z^{2} d z}{\log \left(h_{0} / R\right)}
$$

and

$$
I_{2}=2 \pi R \tau_{0} \int_{z=0}^{z=z_{0}} \frac{z\left(h_{0} / R-1\right) d z}{\log \left(h_{0} / R\right)} .
$$

Following Eq. (6), $\log \left(h_{0} / R\right)$ is replaced by $[1$ $\left.+W_{0}(a z+b)\right]$ and therefore, a change of variable involving $w$ $=W_{0}(a z+b)$ or equivalently $z=\left(w e^{w}-b\right) / a$ is used to rearrange $I_{1}$ and $I_{2}$ such as

$$
I_{1}=\frac{\pi \eta_{B} \dot{\gamma}: \mathbf{p p}}{a^{3}} \int_{-1}^{W_{0}\left(a z_{0}+b\right)}\left(w e^{w}-b\right)^{2} e^{w} d w
$$

and

$$
I_{2}=\frac{2 \pi R \tau_{0}}{a^{2}} \int_{-1}^{W_{0}\left(a z_{0}+b\right)}\left(w e^{w}-b\right)\left(e^{w+1}-1\right) e^{w} d w .
$$

Hence, integrations can be performed more easily and yields after some simplifications

$$
\begin{aligned}
I_{1}= & \frac{\pi \eta_{B} \dot{\gamma}: \mathbf{p p}}{a^{3}}\left[\frac{1}{27}\left(2 e^{3 w}-6 w e^{3 w}+9 w^{2} e^{3 w}\right)\right. \\
& \left.-\frac{e^{2 w-1}}{2}+w e^{2 w-1}+e^{w-2}\right]_{-1}^{W_{0}\left(a z_{0}+b\right)}
\end{aligned}
$$

and

$$
\begin{aligned}
I_{2}= & -\frac{2 \pi R \tau_{0}}{a^{2}}\left[\frac{e^{3 w+1}}{9}+\frac{w}{2} e^{2 w}-\frac{3}{4} e^{2 w}\right. \\
& \left.-\frac{w}{3} e^{3 w+1}+e^{w-1}\right]_{-1}^{W_{0}\left(a z_{0}+b\right)} .
\end{aligned}
$$

And, by continuing the calculation with the fact that $W_{0}\left(a z_{0}+b\right)$ has been replaced by $W_{0}$ and $a z_{0}+b$ by $J$ $=\frac{h}{e R}[\log (h / R)-1]$, one obtains

$$
I_{1}=\pi R^{3} \tau_{0} B_{n}^{2} \frac{\tilde{\dot{\gamma}}: \mathbf{p p}}{|\tilde{\gamma}: \mathbf{p p}|^{3}} A_{1}
$$

where $A_{1}=\left[-\frac{28}{27}+\frac{8 e^{3}}{27}\left(2 \frac{J^{3}}{W_{0}^{3}}-6 \frac{J^{3}}{W_{0}^{2}}+9 \frac{J^{3}}{W_{0}}\right)-4 e^{2} \frac{J^{2}}{W_{0}^{2}}+8 e^{2} \frac{J^{2}}{W_{0}}\right.$ $\left.+8 e \frac{J}{W_{0}}\right]$, and

$$
I_{2}=\pi R^{3} \tau_{0} B_{n}^{2} \frac{1}{|\tilde{\gamma}: \mathbf{p p}|^{2}} A_{2}
$$

where $A_{2}=\left[\frac{14}{9}-\frac{8 e^{3}}{9} \frac{J^{3}}{W_{0}^{3}}-4 e^{2} \frac{J^{2}}{W_{0}}+6 e^{2} \frac{J^{2}}{W_{0}^{2}}+\frac{8 e^{3}}{3} \frac{J^{3}}{W_{0}^{2}}-8 e \frac{J}{W_{0}}\right]$. The above results can be summed up to give the expression for $I$,

$$
I=\pi R^{3} \tau_{0} B_{n}^{2}\left(A_{1} \frac{\tilde{\dot{\gamma}}: \mathbf{p p}}{|\tilde{\dot{\gamma}}: \mathbf{p p}|^{3}}+A_{2} \frac{1}{|\tilde{\dot{\gamma}}: \mathbf{p p}|^{2}}\right) .
$$


Integrands involved in Eq. (A2) can be performed more easily as $h$ is independent of $z$. Hence, Eq. (A2) is found to be

$$
\begin{aligned}
J= & \frac{\pi \eta_{B}}{\log (h / R)} \dot{\gamma}: \mathbf{p p}\left[\frac{L^{3}}{24}-\frac{z_{0}^{3}}{3}\right] \\
& +2 \pi R \tau_{0} \frac{(h / R-1)}{\log (h / R)}\left[\frac{L^{2}}{8}-\frac{z_{0}^{2}}{2}\right] .
\end{aligned}
$$

By noting that $z_{0}=D B_{n}(1+e J) /|\tilde{\dot{\gamma}}: \mathbf{p p}|, J$ becomes

$$
\begin{aligned}
J= & \frac{\pi \eta_{B} L^{3}}{24 \log (h / R)} \dot{\gamma}: \mathbf{p p}+\pi R L^{2} \tau_{0} \frac{(h / R-1)}{\log (h / R)} \\
& -\frac{\pi L^{3} \tau_{0} B_{n}^{2}}{8 a_{r}^{3}}\left(B_{1} \frac{\tilde{\gamma}: \mathbf{p p}}{|\tilde{\dot{\gamma}}: \mathbf{p p}|^{3}}+B_{2} \frac{1}{|\tilde{\gamma}: \mathbf{p p}|^{2}}\right),
\end{aligned}
$$

where $B_{1}=8(1+e J)^{3} / 3 \log (h / R)$ and $B_{2}=4(1+e J)^{2}(h /$ $R-1) / \log (h / R)$.

Finally, the rod stress tensor given by Eq. (21) can be rewritten as

$$
\tau^{p}=\frac{2 \phi}{\pi R^{2} L}\langle\mathbf{p p} I+\mathbf{p p} J\rangle,
$$

which yields after the substitutions of $I$ and $J$

$$
\begin{aligned}
\tau^{p}= & \eta_{B} \phi \frac{a_{r}^{2}}{3 \log (h / R)} \dot{\gamma}:\langle\mathbf{p p p p}\rangle+\tau_{0} \phi \frac{a_{r}(h / R-1)}{\log (h / R)}\langle\mathbf{p p}\rangle \\
& +\tau_{0} \phi \frac{B_{n}^{2}}{a_{r}}\left[\left(A_{1}-B_{1}\right)\left\langle\frac{\tilde{\dot{\gamma}}: \mathbf{p p p p}}{|\tilde{\dot{\gamma}}: \mathbf{p p}|^{3}}\right\rangle\right. \\
& \left.+\left(A_{2}-B_{2}\right)\left\langle\frac{\mathbf{p p}}{|\tilde{\gamma}: \mathbf{p p}|^{2}}\right\rangle\right] .
\end{aligned}
$$

By using an abbreviated notation for the last term in Eq. (A15), the above equation becomes

$$
\begin{aligned}
\tau^{p}= & \eta_{B} \phi \frac{a_{r}^{2}}{3 \log (h / R)} \dot{\gamma}:\langle\mathbf{p p p p}\rangle+\tau_{0} \phi \frac{a_{r}(h / R-1)}{\log (h / R)}\langle\mathbf{p p}\rangle \\
& +\tau_{0} \phi \frac{B_{n}^{2}}{a_{r}}\langle\Theta(\tilde{\gamma}, \mathbf{p}) \mathbf{p p}\rangle,
\end{aligned}
$$

where $\Theta(\tilde{\gamma}, \mathbf{p})$ is a function of the dimensionless strain-rate tensor, $\tilde{\gamma}$, and the unit vector directed along the main particle axis, $\mathbf{p}$.

\section{APPENDIX B: INTEGRATION CALCULATIONS INVOLVED FOR $B_{n} \gg 1$}

The integration in Eq. (23) means to determine $I$ given in Eq. (A1) by replacing the upper limit with $z=L / 2$. Thus, in what follows, $I$ will be denoted by $I^{\prime}$. Thanks to the change of variable used to express $I_{1}$ and $I_{2}$ in Eqs. (A5) and (A6), respectively, the upper limit becomes

$$
W_{0}(a L / 2+b)=W_{0}\left[\frac{1}{e}\left(\frac{a_{r}|\tilde{\gamma}: \mathbf{p p}|}{2 B_{n}}-1\right)\right] .
$$

By using the fact that $B_{n} \gg 1$ and $a_{r} \gg 1$, we can rewrite Eq. (B1) as $W_{K}=W_{0}(K)$, where $K=|\tilde{\gamma}: \mathbf{p p}| / 2 e$. Hence, the integration in Eq. (23) is found to be

$$
I^{\prime}=\pi R^{3} \tau_{0} B_{n}^{2}\left(A_{1}^{\prime} \frac{\tilde{\dot{\gamma}}: \mathbf{p p}}{|\tilde{\dot{\gamma}}: \mathbf{p p}|^{3}}+A_{2}^{\prime} \frac{1}{|\tilde{\dot{\gamma}}: \mathbf{p p}|^{2}}\right),
$$

where $A_{1}^{\prime}=\left[-\frac{28}{27}+\frac{8 e^{3}}{27}\left(2 \frac{K^{3}}{W_{K}^{3}}-6 \frac{K^{3}}{W_{K}^{2}}+9 \frac{K^{3}}{W_{K}}\right)-4 e^{2} \frac{K^{2}}{W_{K}^{2}}\right.$ $\left.+8 e^{2} \frac{K^{2}}{W_{K}}+8 e \frac{K}{W_{K}}\right]$ and $A_{2}^{\prime}=\left[\frac{14}{9}-\frac{8 e^{3}}{9} \frac{K^{3}}{W_{K}^{3}}-4 e^{2} \frac{K^{2}}{W_{K}}+6 e^{2} \frac{K^{2}}{W_{K}^{2}}\right.$ $\left.+\frac{8 e^{3}}{3} \frac{K^{3}}{W_{K}^{2}}-8 e \frac{K}{W_{K}}\right]$. Thus, after substituting $I^{\prime}$ in the definition for the particle stress tensor, one obtains

$$
\begin{aligned}
\tau^{p}= & \eta_{B} \phi \frac{a_{r}^{2}}{3 \log (h / R)} \dot{\boldsymbol{\gamma}}:\langle\mathbf{p p p p}\rangle+\tau_{0} \phi \frac{a_{r}(h / R-1)}{\log (h / R)}\langle\mathbf{p p}\rangle \\
& +\tau_{0} \phi \frac{B_{n}^{2}}{a_{r}}\langle\Phi(\tilde{\gamma}, \mathbf{p}) \mathbf{p p}\rangle,
\end{aligned}
$$

where $\Phi(\tilde{\gamma}, \mathbf{p})$ is a function of the dimensionless strain-rate tensor, $\tilde{\gamma}$, and the unit vector, $\mathbf{p}$.

\section{APPENDIX C: DERIVATION FOR THE EQUATION OF CHANGE OF 〈pp〉}

In this part, we would like to show that Eq. (30a), expressing the time evolution for the second-order orientation $\langle\mathbf{p p}\rangle$, can also be obtained by considering the dynamics for a single rod given by Eq. (31a). Hence, by extending the upper convected derivative for each components of $\langle\mathbf{p p}\rangle$, one gets

$$
\frac{\mathfrak{D}\langle\mathbf{p} \mathbf{p}\rangle}{\mathfrak{D} t}=\left\langle\frac{\mathfrak{D} \mathbf{p}}{\mathfrak{D} t} \mathbf{p}\right\rangle+\left\langle\mathbf{p} \frac{\mathfrak{D} \mathbf{p}}{\mathfrak{D} t}\right\rangle
$$

The substitution of Eq. (31a) in Eq. (C1) results in

$$
\frac{\mathfrak{D}\langle\mathbf{p p}\rangle}{\mathfrak{D} t}=\langle-\dot{\gamma}: \mathbf{p p p}\rangle-\tau_{0} \frac{(h / R-1)}{2 a_{r} \eta_{B}}\left\langle\frac{\partial \psi_{\mathbf{p}}}{\psi_{\mathbf{p}} \partial \mathbf{p}} \mathbf{p}+\mathbf{p} \frac{\partial \psi_{\mathbf{p}}}{\psi_{\mathbf{p}} \partial \mathbf{p}}\right\rangle \text {. }
$$

As $\dot{\gamma}$ is not a function of $\mathbf{p}$, it can be brought outside the averaging brackets. Furthermore, by using the following formulas, $\left\langle\frac{\partial \psi_{\mathbf{p}}}{\psi_{\mathbf{p}} \partial \mathbf{p}} \mathbf{p}\right\rangle=\left\langle\mathbf{p} \frac{\partial \psi_{\mathbf{p}}}{\psi_{\mathbf{p}} \partial \mathbf{p}}\right\rangle=3\langle\mathbf{p} \mathbf{p}\rangle-\delta,{ }^{45}$ one obtains

$$
\frac{\mathfrak{D}\langle\mathbf{p p}\rangle}{\mathfrak{D} t}=-\dot{\gamma}:\langle\mathbf{p p p p}\rangle+\tau_{0} \frac{(h / R-1)}{a_{r} \eta_{B}}(\boldsymbol{\delta}-3\langle\mathbf{p p}\rangle),
$$

which is exactly Eq. (30a).

${ }^{1}$ J. Thomasset, P. Carreau, B. Sanschagrin, and G. Ausias, "Rheological properties of long glass fiber filled polypropylene," J. Non-Newtonian Fluid Mech. 125, 25-34 (2005).

${ }^{2}$ G. Natale, M. C. Heuzey, P. J. Carreau, G. Ausias, and J. Férec, "Rheological modeling of carbon nanotube suspensions with rod-rod interactions," AIChE J. 60, 1476-1487 (2014).

${ }^{3}$ S. Ookawara, A. Yano, K. Ogawa, and K. Taniguchi, "Estimate of red-cell deformability and plasma viscosity based on flow curve," AIChE J. 47, 230-239 (2001).

${ }^{4}$ L. Martinie, P. Rossi, and N. Roussel, "Rheology of fiber reinforced cementitious materials: Classification and prediction," Cem. Concr. Res. 40, 226-234 (2010)

${ }^{5}$ N. Ouari, A. Kaci, A. Tahakourt, and M. Chaouche, "Rheological behaviour of fibre suspensions in non-Newtonian fluids," Appl. Rheol. 21, 54801 (2011)

${ }^{6}$ P. Coussot, H. Tabuteau, X. Chateau, L. Tocquer, and G. Ovarlez, “Aging and solid or liquid behavior in pastes," J. Rheol. 50, 975-994 (2006).

${ }^{7}$ L. Ferrara, Y.-D. Park, and S. P. Shah, "A method for mix-design of fiber-reinforced self-compacting concrete," Cem. Concr. Res. 37, 957-971 (2007). 
${ }^{8}$ L. Ferrara, Y.-D. Park, and S. P. Shah, "Correlation among fresh state behavior, fiber dispersion, and toughness properties of SFRCs," J. Mater. Civ. Eng. 20, 493-501 (2008).

${ }^{9}$ R. Deeb, A. Ghanbari, and B. L. Karihaloo, "Development of selfcompacting high and ultra high performance concretes with and without steel fibres," Cem. Concr. Compos. 34, 185-190 (2012).

${ }^{10}$ R. Deeb, B. L. Karihaloo, and S. Kulasegaram, "Reorientation of short steel fibres during the flow of self-compacting concrete mix and determination of the fibre orientation factor," Cem. Concr. Res. 56, 112-120 (2014).

${ }^{11}$ O. Svec, J. Skocek, H. Stang, M. R. Geiker, and N. Roussel, "Free surface flow of a suspension of rigid particles in a non-Newtonian fluid: A lattice Boltzmann approach," J. Non-Newtonian Fluid Mech. 179-180, 32-42 (2012).

${ }^{12}$ F. Chalencon, L. Orgeas, P. J. J. Dumont, G. Foray, J. Y. Cavaille, E. Maire, and S. R. du Roscoat, "Lubricated compression and X-ray microtomography to analyse the rheology of a fibre-reinforced mortar," Rheol. Acta 49, 221235 (2010).

${ }^{13}$ B. Derakhshandeh, S. G. Hatzikiriakos, and C. P. J. Bennington, "Rheology of pulp suspensions using ultrasonic Doppler velocimetry," Rheol. Acta 49, 1127-1140 (2010).

${ }^{14}$ B. Derakhshandeh, S. G. Hatzikiriakos, and C. P. J. Benningtonb, "The apparent yield stress of pulp fiber suspensions," J. Rheol. 54, 1137-1154 (2010).

${ }^{15}$ S.-T. Kang and J.-K. Kim, "Numerical simulation of the variation of fiber orientation distribution during flow molding of ultra high performance cementitious composites (UHPCC)," Cem. Concr. Compos. 34, 208-217 (2012).

${ }^{16} \mathrm{G}$. Jeffery, "The motion of ellipsoidal particles immersed in a viscous fluid," Proc. R. Soc. A 102, 161-179 (1922).

${ }^{17}$ J. Jarrin, E. Vinciguerra, G. Ausias, M. Vincent, and F. Dawans, "Extrusion of tubes of fiber-reinforced thermoplastics," U.S. patent 5307843 (3 May 1994).

${ }^{18}$ A. Poitou, F. Chinesta, and G. Bernier, "Orienting fibers by extrusion in reinforced reactive powder concrete," J. Eng. Mech. 127, 593-598 (2001).

${ }^{19} \mathrm{X}$. Zhou and Z. Li, "Numerical simulation of ram extrusion in short-fiberreinforced fresh cementitious composites," J. Mech. Mater. Struct. 4, 17551769 (2009)

${ }^{20} \mathrm{G}$. K. Batchelor, "The stress system in a suspension of force-free particles," J. Fluid Mech. 41, 545-570 (1970).

${ }^{21}$ E. J. Hinch and L. G. Leal, "Constitutive equations in suspension mechanics. Part 1. General formulation,” J. Fluid Mech. 71, 481-495 (1975).

${ }^{22}$ S. M. Dinh and R. C. Armstrong, "A rheological equation of state for semiconcentrated fiber suspensions," J. Rheol. 28, 207-227 (1984).

${ }^{23}$ G. L. Hand, "A theory of dilute suspensions," Arch. Ration. Mech. Anal. 7, 81-86 (1961).

${ }^{24} \mathrm{H}$. Giesekus, "Elasto-viskose flüssigkeiten, für die in stationären schichtströmungen sämtliche normalspannungskomponenten verschieden groß sind," Rheol. Acta 2, 50-62 (1962).

${ }^{25}$ G. G. Lipscomb, M. M. Denn, D. U. Hur, and D. V. Boger, "The flow of fiber suspensions in complex geometries," J. Non-Newtonian Fluid Mech. 26, 297-325 (1988).

${ }^{26}$ A. G. Gibson and S. Toll, "Mechanics of the squeeze flow of planar fibre suspensions," J. Non-Newtonian Fluid Mech. 82, 1-24 (1999).

${ }^{27}$ B. Souloumiac and M. Vincent, "Steady shear viscosity of short fibre suspensions in thermoplastics," Rheol. Acta 37, 289-298 (1998).

${ }^{28}$ G. K. Batchelor, "The stress generated in a non-dilute suspension of elongated particles by pure straining motion,” J. Fluid Mech. 46, 813-829 (1971).

${ }^{29}$ L. G. Leal, "The slow motion of slender rod-like particles in a second-order fluid," J. Fluid Mech. 69, 305-337 (1975).
${ }^{30} \mathrm{P}$. Brunn, "Interaction of spheres in a viscoelastic fluid," Rheol. Acta 16, 461-475 (1977).

${ }^{31}$ C. Kagarise, K. Miyazono, M. Mahboob, K. W. Koelling, and S. E. Bechtel, "A constitutive model for characterization of shear and extensional rheology and flow induced orientation of carbon nanofiber/polystyrene melt composites," J. Rheol. 55, 781-807 (2011).

${ }^{32}$ J. White, K. Kim, and D. Robinson, "Alternate models for a transversely isotropic plastic viscoelastic fluid," J. Non-Newtonian Fluid Mech. 83, 1932 (1999).

${ }^{33}$ D. Robinson, K. Kim, and J. White, "Constitutive model of a transversely isotropic Bingham fluid,” J. Appl. Mech. 69, 641-648 (2002).

${ }^{34}$ J. Férec, A. Perrot, and G. Ausias, "Toward modeling anisotropic yield stress and consistency induced by fiber in fiber-reinforced viscoplastic fluids," $\mathrm{J}$. Non-Newtonian Fluid Mech. 220, 69-76 (2015).

${ }^{35}$ J. D. Goddard, "The stress field of slender particles oriented by a nonNewtonian extensional flow," J. Fluid Mech. 78, 177-206 (1976).

${ }^{36}$ J. D. Goddard, "Tensile stress contribution of flow-oriented slender particles in non-Newtonian fluids," J. Non-Newtonian Fluid Mech. 1, 1-17 (1976).

${ }^{37}$ J. Férec, E. Bertevas, B. C. Khoo, G. Ausias, and N. Phan-Thien, "The effect of shear-thinning behaviour on rod orientation in filled fluids," J. Fluid Mech. 798, 350-370 (2016).

${ }^{38}$ R. B. Bird, R. C. Armstrong, and O. Hassager, Dynamics of Polymeric Liquids: Fluid Mechanics, 2nd ed. (Wiley, New York, 1987), Vol. 1, pp. xxi, 649.

${ }^{39}$ M. Doi and S. F. Edwards, "Dynamics of rod-like macromolecules in concentrated solution. Part 1," J. Chem. Soc., Faraday Trans. 2 74, 560-570 (1978).

${ }^{40}$ M. Doi and S. F. Edwards, "Dynamics of rod-like macromolecules in concentrated solution. Part 2," J. Chem. Soc., Faraday Trans. 2 74, 918-932 (1978).

${ }^{41}$ E. Mitsoulis, "Flows of viscoplastic materials: Models and computations," in Rheology Reviews 2007 (British Society of Rheology, 2007), pp. 135178.

${ }^{42}$ E. Bingham, Fluidity and Plasticity (McGraw-Hill, New York, 1922).

${ }^{43}$ J. G. Oldroyd, "A rational formulation of the equations of plastic flow for a Bingham solid," Math. Proc. Cambridge Philos. Soc. 43, 100-105 (1947).

${ }^{44}$ R. M. Corless, G. H. Gonnet, D. E. G. Hare, D. J. Jeffrey, and D. E. Knuth, "On the Lambert $W$ function," Adv. Comput. Math. 5, 329-359 (1996).

${ }^{45}$ R. B. Bird, C. F. Curtiss, R. C. Armstrong, and O. Hassager, Dynamics of Polymeric Liquids. Volume 2, Kinetic Theory, 2nd ed. (Wiley, New York, 1987), pp. xxi, 437.

${ }^{46} \mathrm{~S}$. G. Advani and C. L. Tucker, "The use of tensors to describe and predict fiber orientation in short fiber composites," J. Rheol. 31, 751-784 (1987).

${ }^{47}$ E. J. Hinch and L. G. Leal, "Constitutive equations in suspension mechanics. Part 2. Approximate forms for a suspension of rigid particles affected by Brownian rotations," J. Fluid Mech. 76, 187-208 (1976).

${ }^{48}$ K. R. J. Ellwood, G. C. Georgiou, T. C. Papanastasiou, and J. O. Wilkes, "Laminar jets of Bingham-plastic liquids," J. Rheol. 34, 787-812 (1990).

${ }^{49}$ F. Folgar and C. L. Tucker, "Orientation behavior of fibers in concentrated suspensions," J. Reinf. Plast. Compos. 3, 98-119 (1984).

${ }^{50} \mathrm{D}$. H. Chung and T. H. Kwon, "Improved model of orthotropic closure approximation for flow induced fiber orientation," Polym. Compos. 22, 636649 (2001).

${ }^{51} \mathrm{~S}$. T. Chung and T. H. Kwon, "Coupled analysis of injection molding filling and fiber orientation, including in-plane velocity gradient effect," Polym. Compos. 17, 859-872 (1996). 